\title{
$\alpha$-spinasterol from Melandrium firmum attenuates benign prostatic hyperplasia in a rat model
}

\author{
MEE-YOUNG LEE ${ }^{1}$, IN-SIK SHIN ${ }^{1}$, HWANGBO KYOUNG ${ }^{2}$, CHANG-SEOB SEO ${ }^{1}$, \\ JONG-KEUN SON ${ }^{2}$ and HYEUN-KYOO SHIN ${ }^{1}$ \\ ${ }^{1}$ Basic Herbal Medicine Research Group, Korea Institute of Oriental Medicine, Yuseung-gu, Daejeon 305-811; \\ ${ }^{2}$ College of Pharmacy, Yeungnam University, Gyeongsan, Gyeongsangbuk-do 712-749, Republic of Korea
}

Received August 1, 2013; Accepted February 28, 2014

DOI: $10.3892 / \mathrm{mmr} .2014 .2081$

\begin{abstract}
Spinasterol, a biologically active compound, exhibits a number of pharmacological activities, including antitumor, antiulcerogenic and anticarcinogenic activity, and originates from the aerial parts of Aster scaber Thunb (Asteraceae). The present study investigated whether $\alpha$-spinasterol isolated from Melandrium firmum Rohrbach could prevent benign prostatic hyperplasia (BPH) induced by testosterone propionate (TP) in rats. Male Wistar rats were randomly divided into four groups of eight rats following castration. A negative control group received subcutaneous injections of corn oil. Treatments were administered orally $1 \mathrm{~h}$ prior to TP injection. All the rats were sacrificed at the scheduled termination time and their prostates were removed, cleaned and weighed. The prostate size ratio (prostate weight/rat body weight) was then calculated. Additional histopathological examinations were conducted, and the levels of TP and dihydrotestosterone (DHT) in the serum and prostate were measured. TP significantly increased the prostate size ratio $(\mathrm{P}<0.01)$, and $\mathrm{DHT}$ and testosterone levels in the serum and prostate. The TP-induced increase was significantly inhibited in $\alpha$-spinasterol-treated rats when compared with the negative controls $(\mathrm{P}<0.05)$. In addition, histopathological examination demonstrated that $\alpha$-spinasterol treatment suppressed TP-induced prostatic hyperplasia. It is concluded that $\alpha$-spinasterol can prevent TP-induced prostatic hyperplasia and may be beneficial in the management of $\mathrm{BPH}$.
\end{abstract}

Correspondence to: Professor Jong-Keun Son, College of Pharmacy, Yeungnam University, 214-1 Daedong, Gyeongsan 712-749, Republic of Korea

E-mail: cozy11@kiom.re.kr

Dr Hyeun-Kyoo Shin, Basic Herbal Medicine Research Group, Korea Institute of Oriental Medicine, 483 Exporo, Daejeon, Yuseung-gu 305-811, Republic of Korea

E-mail: hkshin@kiom.re.kr

Key words: $\alpha$-spinasterol, benign prostatic hyperplasia, testosterone, dihydrotestosterone

\section{Introduction}

Benign prostatic hyperplasia (BPH) is extremely common and it is clinically characterized by prostate enlargement and lower urinary tract symptoms. Despite the enormous burden of $\mathrm{BPH}$ on the public health, the pathogenesis of $\mathrm{BPH}$ is only partially understood $(1,2)$. It is well known that enlargement of the prostate occurs in the presence of androgens (3) and that anabolic steroids increase prostatic volume and reduce urine flow, leading to increased urinary frequency (4). The prostate is an androgen-dependent organ where testosterone and its precursors of extratesticular origin are bioactivated to the more potent dihydrotestosterone (DHT) (5). The prostate gland is generally considered a significant site of DHT formation (6). The systemic effect of endocrine activity in the prostate gland is principally DHT formation and its release to the circulation. The production of DHT increases with age, which induces the enhancement of prostate growth and hyperplasia (7). The significance of DHT is supported by clinical studies where an inhibitor of $5 \alpha$-reductase was administered to males with BPH. In a number of cases, therapy with a $5 \alpha$-reductase inhibitor significantly reduced the DHT level of the prostate and prostate size (8). Finasteride is widely used in the treatment of androgen-dependent diseases, specifically male pattern baldness, BPH and prostate cancer (9). Finasteride is a competitive and specific inhibitor of $5 \alpha$-reductase, and has a role in inhibiting the conversion of testosterone to DHT in androgen-sensitive tissues, including the prostate and hair follicles, and therefore suppressing the serum and intraprostatic DHT concentrations (10). Conventionally, drugs, including finasteride and dutasteride, have been shown to be an effective treatment for $\mathrm{BPH}$, but their use is restricted due to associated side effects, including erectile dysfunction, loss of libido, dizziness and upper respiratory tract infection $(11,12)$.

Spinasterol is a biologically active compound isolated from the aerial parts of Aster scaber Thunb (Asteraceae), which exhibits various pharmacological activities, including antitumor, antiulcerogenic and anticarcinogenic activities (13-15). A study has demonstrated that spinasterol also has anti-inflammatory effects (16). However, there is no previous study of the possible efficacy of using $\alpha$-spinasterol to treat testosterone-induced $\mathrm{BPH}$ model in rats. In the present study, a testosterone-induced rat model of BPH was used $(17,18)$ and the 
therapeutic effects of $\alpha$-spinasterol were evaluated, including its inhibition of the production of androgen hormones, such as testosterone and DHT.

\section{Materials and methods}

Plant materials containing $\alpha$-spinasterol. Whole plants of Melandrium firmum Rohrbach were purchased in March 2005 from a folk medicine market, 'Yak-ryong-si', in Daegu and the material was confirmed taxonomically by Professor Ki-Hwan Bae, of Chungnam National University (Daejeon, Republic of Korea). A voucher specimen (YNS-99-01) was preserved at the College of Pharmacy, Yeungnam University (Gyeongsan, North Gyeongsang).

Instruments and reagents. Melting points were measured using Fisher-Johns melting point apparatus (Thermo Fisher Scientific Co., St. Louis, MO, USA) and they were uncorrected. Fourier transform-infrared spectroscopy (FT-IR) spectra were recorded using a JASCO FT-IR 300E spectrophotometer (Jasco, Tokyo, Japan). Nuclear magnetic resonance (NMR) spectra were recorded with a Bruker $250 \mathrm{MHz}$ (DMX 250) spectrometer (Bruker, Rheinstetten, Germany) using a standard Bruker pulse program. The samples were dissolved in pyridine- $\mathrm{d}_{5}$ and chemical shifts were reported in parts per million downfield of tetramethylsilane. The electrospray ionization (ESI) mass spectrometry (MS) spectrum was measured using an LCQ advantage-trap mass spectrometer (Thermo Finnigan, San Jose, CA, USA) equipped with an ESI source. Stationary phases for column (silica gel 60, 70-230 and 270-400 mesh) and thin layer chromatography plates (silica gel $60 \mathrm{~F}_{254}$ and RP-18 $\mathrm{F}_{254}$ ) were purchased from Merck KGaA (Darmstadt, Germany). Spots were detected under ultraviolet radiation and by spraying with $10 \% \mathrm{H}_{2} \mathrm{SO}_{4}$, followed by heating. All other chemicals and solvents were of analytical grade and used without further purification.

Extraction and isolation. Whole plants of Melandrium firmum Rohrbach $(10.0 \mathrm{~kg})$ were extracted three times with $80 \% \mathrm{MeOH}$ (20 liter) under reflux for $12 \mathrm{~h}$, then filtered and concentrated to yield the $\mathrm{MeOH}$ extract (550.0 g). The $\mathrm{MeOH}$ extract was suspended in $\mathrm{H}_{2} \mathrm{O}$ and extracted with hexane, EtOAc and then $\mathrm{BuOH}$ to yield the hexane- $(75.9 \mathrm{~g})$, EtOAc- (47.4 g), and BuOH-soluble fractions (97.0 g), respectively. The hexane-soluble fraction $(50.0 \mathrm{~g})$ was subjected to column chromatography with silica gel $(60 \times 12 \mathrm{~cm}$, silica gel 70-230 mesh) and eluted using a stepwise gradient of hexane and EtOAc $(100: 1 \rightarrow 1: 1)$ to yield 32 subfractions. Fraction 14 (2.5 g) was loaded onto a silica gel column $(4 \times 50 \mathrm{~cm}$, silica gel 70-230 mesh) and eluted with hexane: EtOAc (50:1-1:1) to give compound $1(300 \mathrm{mg})$. The chemical structure of the isolated compound was determined by the comparison of physicochemical and spectral data with published values.

$\alpha$-spinasterol (1): Colorless crystals, melting point: $171-173^{\circ} \mathrm{C}$; IR $v_{\max }(\mathrm{KBr}) / \mathrm{cm}: 3,251(\mathrm{OH}), 1,676(\mathrm{C}=\mathrm{C}$ stretch), 1,471 $\left(\mathrm{CH}_{2}\right), 1,371\left(\mathrm{CH}_{3}\right) ;{ }^{1} \mathrm{H}-\mathrm{NMR}(250 \mathrm{MHz}$, pyridine- $\left.\mathrm{d}_{5}\right) \delta: 5.18(1 \mathrm{H}, \mathrm{dd}, \mathrm{J}=15.0,8.7 \mathrm{~Hz}, \mathrm{H}-22), 5.14(1 \mathrm{H}$, t, J=4.0 Hz, H-7), 5.08 (1H, dd, J=15.0, 8.7 Hz, H-23), 3.84 (1H, m, H-3), 1.06 (3H, d, J=6.5 Hz, $\left.\mathrm{CH}_{3}-21\right), 0.87$ (3H, d,
$\left.\mathrm{J}=6.8 \mathrm{~Hz}, \mathrm{CH}_{3}-26\right), 0.85\left(3 \mathrm{H}, \mathrm{d}, \mathrm{J}=6.5 \mathrm{~Hz}, \mathrm{CH}_{3}-27\right), 0.82$ (3H, s, $\left.\mathrm{CH}_{3}-19\right), 0.59$ (3H, s, $\left.\mathrm{CH}_{3}-18\right) ;{ }^{13} \mathrm{C}-\mathrm{NMR}(62.5 \mathrm{MHz}$, pyridine- $\left.\mathrm{d}_{5}\right)$ $\delta: 139.6(\mathrm{C}-8), 138.6(\mathrm{C}-22), 129.6$ (C-23), 117.9 (C-7), 71.0 (C-3), 55.9 (C-17), 55.3 (C-14), 51.3 (C-24), 49.7 (C-9), 43.4 (C-13), 41.1 (C-20), 40.5 (C-5), 39.6 (C-12), 38.9 (C-4), 37.6 (C-25), 37.5 (C-1), 34.4 (C-10), 32.1 (C-2), 29.5 (C-6), 28.2 (C-16), 25.6 (C-28), 23.3 (C-15), 21.8 (C-11), 21.5 (C-26), 21.2 (C-21), 19.1 (C-27), 13.2 (C-18), 12.4 (C-29), 12.1 (C-19). Fast atom bombardment-MS m/z $412[\mathrm{M}]^{+}$.

Animals. Male 12-week-old Wistar-Unilever rats weighing 250-350 g (Central Lab. Animal, Inc., Seoul, Korea) were housed in a room maintained at $18-23^{\circ} \mathrm{C}$ and with a relative humidity of $40-60 \%$ and an alternating $12 \mathrm{~h}$ light/dark cycle. The rats were fed a standard laboratory diet with water ad libitum. All experimental procedures were conducted in accordance with the NIH Guidelines for the Care and Use of Laboratory Animals, and animal handling followed the guidelines of the National Animal Welfare Law of Korea.

Castration procedure. Following a week of acclimatization, to prevent the influence of intrinsic testosterone, rats in all groups were castrated three days prior to the beginning of the experiments. All the animals were anesthetized by intraperitoneal injection of pentobarbital. Castration involved removal of the testicles and the epididymal fat through the scrotal sac, following a previously described method (19). The spermatic cord and blood vessels were ligated with 3-0 sutures and resected.

Induction of $\mathrm{BPH}$ and treatments. The rats were randomly divided into five groups $(n=8)$ following castration and a week of acclimatization. Experimental groups 2-5 had BPH induced via daily subcutaneous injections for four weeks with testosterone propionate (TP; $3 \mathrm{mg} / \mathrm{kg}$ ) dissolved in corn oil. The animals in the experimental groups received phosphate-buffered saline (PBS), finasteride (positive control, $10 \mathrm{mg} / \mathrm{kg}$ ), and $\alpha$-spinasterol ( $5 \mathrm{mg} / \mathrm{kg}$ ) by gavage during the four weeks of TP injection. The negative control groups were orally administered PBS and received daily subcutaneous injections of corn oil for four weeks. Finasteride was used for the treatment of $\mathrm{BPH}$, since it is a well-known $5 \alpha$-reductase inhibitor. The effective dose of finasteride was based on previous reports. The body weight was measured weekly during the experiment. The application volume was calculated in advance, based on the most recently recorded body weight of individual animals.

Following the final treatment and overnight fasting, all animals were anesthetized with pentobarbital. The blood samples were drawn from the caudal vena cava. The serum was separated by centrifugation for $10 \mathrm{~min}$ at $200 \mathrm{x} \mathrm{g}$ and stored at $-80^{\circ} \mathrm{C}$. Whole prostates were immediately removed, weighed and their relative organ weight was calculated as the ratio of prostate to body weight. The percentage of inhibition of prostate to relative organ weight by $\alpha$-spinasterol was calculated as previously described (20). Sections of the ventral prostate lobe were fixed with $10 \%$ buffered formalin and embedded in paraffin for histological analysis. The remaining sections of the prostates were stored at $-80^{\circ} \mathrm{C}$ to evaluate hormone levels. 
Homogenate preparation. Prostate tissue was homogenized $(1 / 10 \mathrm{w} / \mathrm{v})$ in a tissue lysis/extraction reagent containing protease inhibitors (Sigma, St. Louis, MO, USA) using a homogenizer (IKA, Staufen, Germany). Homogenates were centrifuged at $12,000 \mathrm{x} \mathrm{g}$ for $25 \mathrm{~min}$ at $4^{\circ} \mathrm{C}$, and protein concentrations in the supernatant fractions were determined using a Bradford reagent (Bio-Rad, Hercules, CA, USA).

Measurements of testosterone and DHT in serum and prostate. The levels of testosterone and DHT in the serum and prostate were measured by an enzyme-linked immunosorbent assay. DHT kits were purchased from ALPCO Diagnostics (Salem, NH, USA) and testosterone kits were obtained from Cayman (Ann Harbor, MI, USA). The values are expressed per $\mathrm{mg}$ protein in the prostate and per $\mathrm{ml}$ in the serum.

Histopathological examination. To assess morphological changes in the prostate, the tissue was embedded in paraffin, cut into $4-\mu \mathrm{m}$ thick sections and stained with hematoxylin and eosin solution (hematoxylin, Sigma MHS-16, and eosin, Sigma HT110-1-32). The tissues were mounted, placed under a coverslip using Dako mounting medium (Dakocytomation, Denmark, CA, USA) and examined microscopically (Olympus BX51; Olympus, Tokyo, Japan).

Statistical analysis. Data are expressed as the mean \pm standard deviation. Statistical significance was determined using Student's two-tailed t-test to compare independent means with Microsoft Excel (Microsoft, London, UK). $\mathrm{P}<0.01$ or $\mathrm{P}<0.05$ were considered to indicate a statistically significant difference.

\section{Results}

Effect of $\alpha$-spinasterol on prostate weight. The relative prostate weights of TP-induced BPH animals were significantly higher compared with the negative control group. The body weight of animals treated with $\alpha$-spinasterol was not significantly different from that of BPH animals (Fig. 1A). However, a significant reduction in the relative prostate weight was identified in animals treated with $\alpha$-spinasterol when compared with the TP-induced BPH group (Fig. 1B).

Effects of a-spinasterol on testosterone and DHT levels in the serum. The TP-induced BPH animals had significantly higher serum testosterone levels $(20.80 \pm 2.63 \mathrm{ng} / \mathrm{ml})$ compared with the negative controls $(0.14 \pm 0.18 \mathrm{ng} / \mathrm{ml})$. However, animals treated with $\alpha$-spinasterol $(10.53 \pm 2.39 \mathrm{ng} / \mathrm{ml}, \mathrm{P}<0.05)$ had significantly lower serum testosterone levels compared with TP-induced BPH animals (Fig. 2A). The DHT level in animals treated with $\alpha$-spinasterol $(14.74 \pm 2.38 \mathrm{ng} / \mathrm{ml}, \mathrm{P}<0.05)$ was also significantly lower compared with TP-induced BPH animals $(23.95 \pm 3.74 \mathrm{ng} /$ $\mathrm{ml}, \mathrm{P}<0.01$ ) (Fig. 2B).

Effect of $\alpha$-spinasterol on testosterone and DHT levels in the prostate. The TP-induced BPH animals had higher levels of prostate testosterone $(3.25 \pm 0.23 \mathrm{ng} / \mathrm{ml}, \mathrm{P}<0.01)$ and DHT (426.01 $\pm 144.05 \mathrm{pg} / \mathrm{ml}, \mathrm{P}<0.01)$ compared with the castrated group. The group treated with finasteride had significantly lower testosterone levels $(1.87 \pm 0.27 \mathrm{ng} / \mathrm{ml}, \mathrm{P}<0.01)$ and DHT levels

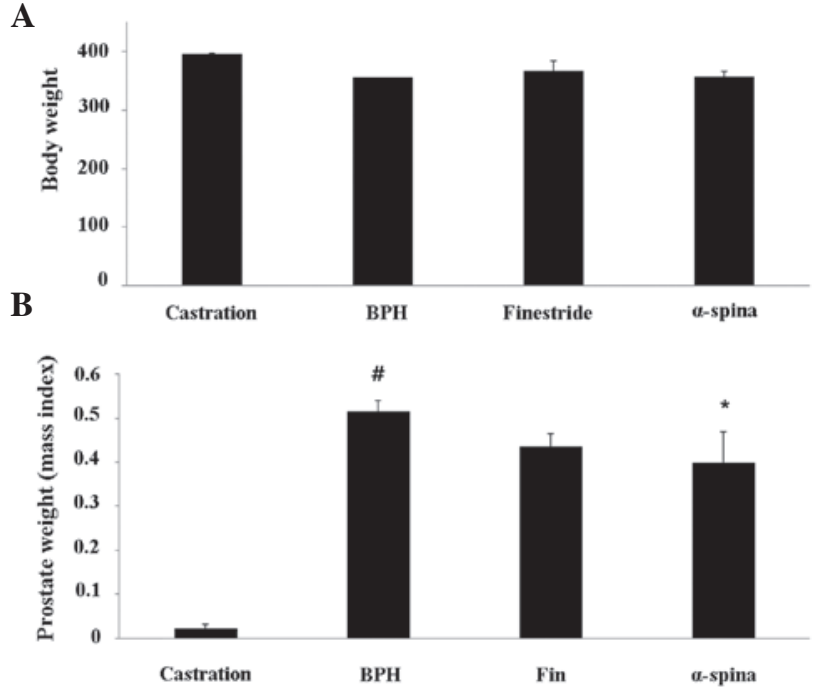

Figure 1. Effects of $\alpha$-spinasterol on (A) body and (B) prostate weights in testosterone propionate-treated rats. Castration, corn oil injection (s.c.) + PBS (p.o.); BPH, testosterone (s.c.) + PBS (p.o.); finasteride, finasteride $(10 \mathrm{mg} / \mathrm{kg}$, p.o.) + testosterone (s.c.); $\alpha$-spinasterol, $\alpha$-spinasterol $(5 \mathrm{mg} / \mathrm{kg}, \mathrm{p} . \mathrm{o})+$. testosterone (s.c.). $\alpha$-spinasterol and finasteride treatments were performed $1 \mathrm{~h}$ prior to testosterone injection. ${ }^{\#} \mathrm{P}<0.05$ vs. the castration group. ${ }^{*} \mathrm{P}<0.05$ vs. the BPH group. PBS, phosphate-buffered saline; p.o., peroral; $\mathrm{BPH}$, benign prostatic hyperplasia; s.c., subcutaneously.
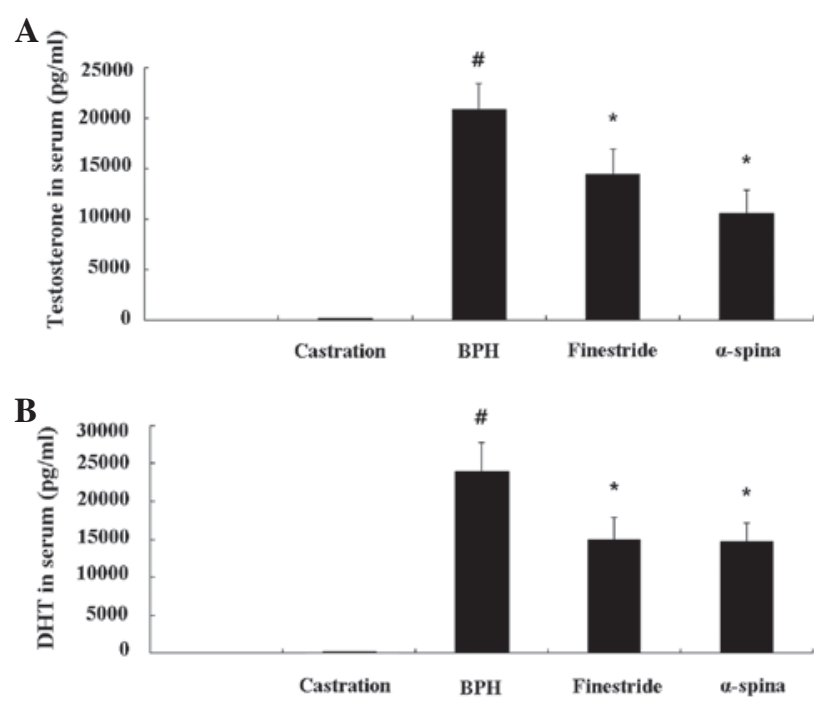

Figure 2. Effects of $\alpha$-spinasterol on (A) testosterone and (B) DHT levels in serum. The levels of testosterone and DHT were significantly lower in the serum of the $\alpha$-spinasterol treatment group compared with the BPH group. Castration, corn oil injection (s.c.) + PBS (p.o.); BPH, testosterone (s.c.) + PBS (p.o.); finasteride, finasteride (10 mg/kg, p.o.) + testosterone (s.c.); $\alpha$-spinasterol, $\alpha$-spinasterol (5 mg/kg, p.o.) + testosterone (s.c.). $\alpha$-spinasterol and finasteride treatments were performed $1 \mathrm{~h}$ prior to testosterone injection. ${ }^{\#} \mathrm{P}<0.05$ vs. the castration group. ${ }^{*} \mathrm{P}<0.05$ level vs. the $\mathrm{BPH}$ group. DHT, dihydrotestosterone; $\mathrm{BPH}$, benign prostatic hyperplasia; PBS, phosphate-buffered saline; p.o, paroral; s.c., subcutaneously.

$(126.22 \pm 34.66 \mathrm{pg} / \mathrm{ml}, \mathrm{P}<0.01)$ compared with $\mathrm{BPH}$ animals. As with the finasteride-treated group, animals treated with $\alpha$-spinasterol had significantly lower testosterone $(1.47 \pm 0.27 \mathrm{ng} /$ $\mathrm{ml}, \mathrm{P}<0.01)$ and DHT levels $(133.31 \pm 35.27 \mathrm{pg} / \mathrm{ml}, \mathrm{P}<0.01)$ compared with BPH animals (Fig. 3A and 3B). 

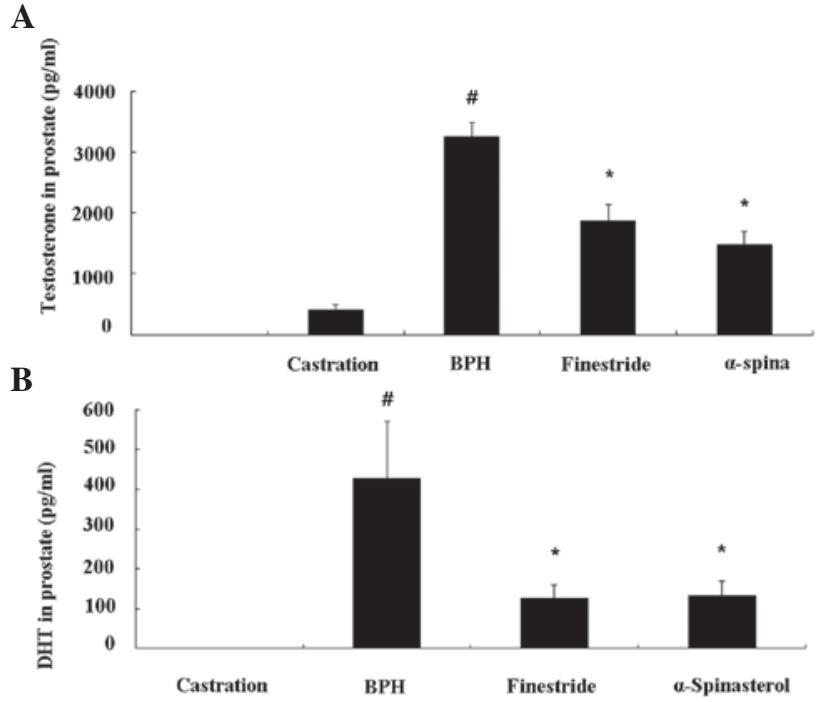

Figure 3. Effects of $\alpha$-spinasterol on (A) testosterone and (B) DHT levels in the prostate. The levels of testosterone and DHT in the prostate were significantly lower in the $\alpha$-spinasterol treatment group compared with the BPH group. Castration, corn oil injection (s.c.) + PBS (p.o.); BPH, testosterone (s.c.) + PBS (p.o.); Finasteride, finasteride $(10 \mathrm{mg} / \mathrm{kg}$, p.o.) + testosterone (s.c.) $\alpha$-spinasterol, $\alpha$-spinasterol (5 mg/kg, p.o.) + testosterone (s.c.). $\alpha$-spinasterol and finasteride treatments were performed $1 \mathrm{~h}$ prior to testosterone injection. ${ }^{\#} \mathrm{P}<0.05$ vs. the castration group. ${ }^{*} \mathrm{P}<0.05$ vs. the BPH group. DHT, dihydrotestosterone; PBS, phosphate-buffered saline; BPH, benign prostatic hyperplasia; p.o., paroral; s.c., subcutaneously.

Effects of $\alpha$-spinasterol on the histomorphology of prostate tissue. The epithelial cell layers of the prostate were larger in TP-induced BPH animals compared with the negative controls. Notably, this histological analysis revealed that administration of $\alpha$-spinasterol marginally altered the histoarchitecture of rats with BPH (Fig. 4).

\section{Discussion}

In the present study, a rat model was used to examine the effect of $\alpha$-spinasterol isolated from Melandrium firmum Rohrbach on the progression of induced prostatic epithelial hyperplasia. The results demonstrated that relative prostate weight, androgen (DHT and testosterone) levels in prostate and serum, and histopathological changes associated with prostatic hyperplasia were significantly reduced by administration of $\alpha$-spinasterol.

The reduction of prostate weight following the administration of $\alpha$-spinasterol in the present study may be due to decreased levels of DHT and testosterone. According to previous studies, testosterone and DHT exhibit key role in the development of the male reproductive organs, and these hormones are commonly associated with $\mathrm{BPH}(21,22)$. Aging leads to excessive production of DHT, which causes the development and exacerbation of BPH (21). The relative prostatic weight of animals with BPH is significantly increased compared with a vehicle control, whereas the relative prostatic weight is reduced in animals treated with finasteride or lauric/myristic acid (20).

The results of the present study were consistent with the histopathological examination of prostate tissue. BPH
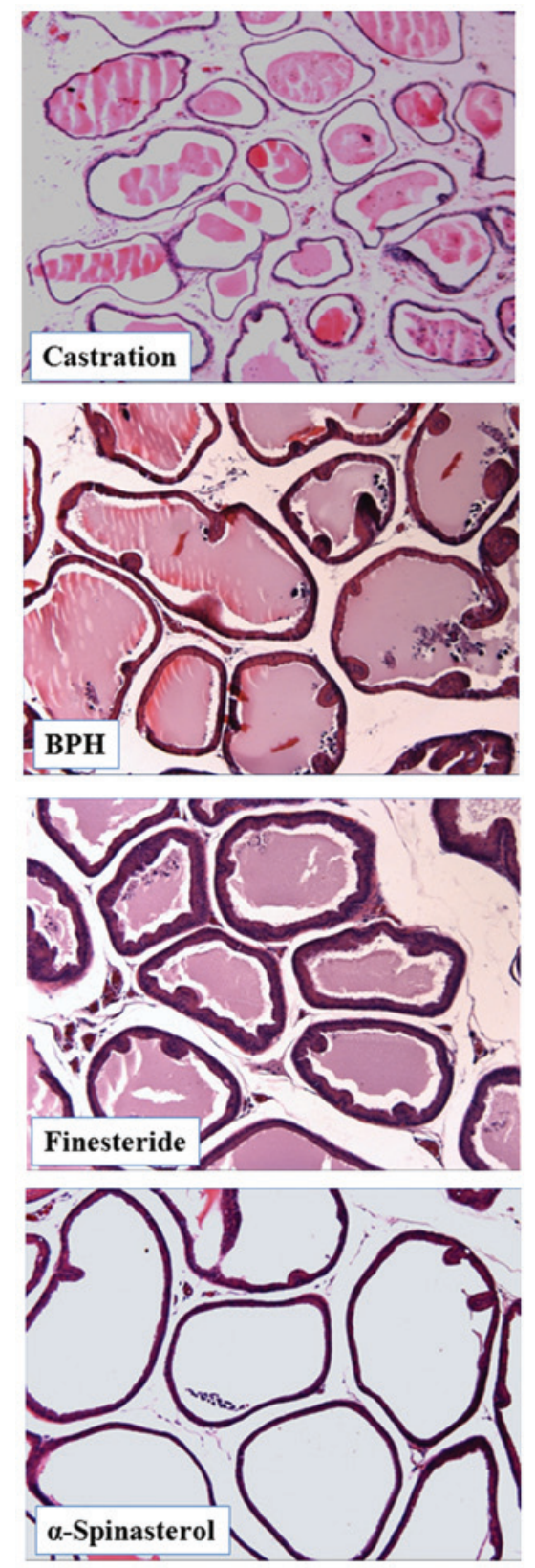

Figure 4. Effects of $\alpha$-spinasterol on prostate hyperplasia. Histological examination of prostate tissue was performed $24 \mathrm{~h}$ after the final testosterone injection. Prostate tissues were fixed, sectioned at a $4-\mu \mathrm{m}$ thickness, and stained with hematoxylin and eosin solution (magnification, x200). BPH, benign prostatic hyperplasia.

animals exhibited glandular hyperplasia in the prostate, whereas animals treated with $\alpha$-spinasterol demonstrated only mild glandular hyperplasia. Therefore, these findings and the results of the hormone examination indicate that $\alpha$-spinasterol is effective in the treatment of BPH.

$\alpha$-spinasterol also decreased the levels of testosterone and DHT in serum and prostate gland. These findings, and the results of the relative weight and histopathological examination of prostates, indicate that $\alpha$-spinasterol inhibits the development of BPH in rats, and these effects are closely associated with a reduction in DHT level. Therefore, higher serum and prostate DHT levels may be associated with a larger prostate volume and a higher prevalence of $\mathrm{BPH}$. 
In conclusion, oral administration of $\alpha$-spinasterol in a $\mathrm{BPH}$ rat model significantly decreased prostate size, prostatic hyperplasia and DHT levels in the serum and prostate. This information may provide an improved understanding of the pathogenesis of age-dependent conditions, including BPH. In addition, it may aid in the identification of improved targets and the development of more specific therapeutic agents to treat prostate conditions. The results of the present study indicate that $\alpha$-spinasterol may be a useful agent in BPH treatment.

\section{Acknowledgements}

This study was supported by an 'Evidence-based medicine for herbal formulae' grant from the Korea Institute of Oriental Medicine.

\section{References}

1. Parsons JK and Kashefi C: Physical activity, benign prostatic hyperplasia, and lower urinary tract symptoms. EurUrol 53: 1228-1235, 2008.

2. Roehrborn CG, Siami P, Barkin J, Damião R, Gecher E, Miñana B, Mirone V, Castro R, Wilson T and Montorsi F: The influence of baseline parameters on changes in international prostate symptom score with dutasteride, tamsulosin, and combination therapy among men with symptomatic benign prostatic hyperplasia and an enlarged prostate: 2 -year data from the CombAT study. Eur Urol 55: 461-471, 2009.

3. McConnell JD: Benign prostatic hyperplasia. Hormonal treatment. Urol Clin North Am 22: 387-400, 1995.

4. Wemyss-Holden SA, Hamdy FC and Hastie KJ: Steroid abuse in athletes, prostatic enlargement and bladder outflow obstruction - is there a relationship? Br J Urol 74: 476-478, 1994.

5. Bruchovsky N, Rennie PS, Batzold FH, Goldenberg SL, Fletcher T and McLoughlin MG: Parameters of 5 alpha-reductase activity in stroma and epithelium of normal, hyperplastic, and carcinomatous human prostates. J Clin Endocrinol Metab 67: 806-816, 1998.

6. Thigpen AE, Silver RI, Guileyardo JM, Casey ML, McConnell JD and Russell DW: Tissue distribution and ontogeny of steroid 5 alpha-reductase isozyme expression. J Clin Invest 92: 903-910, 1993.

7. Carson C III and Rittmaster R: The role of dihydrotestosterone in benign prostatic hyperplasia. Urology 61 (4 Suppl 1): 2-7, 2003.
8. Kumar VL and Wahane VD: Current status of $5 \alpha$-reductase inhibitors in the treatment of benign hyperplasia of prostate. Indian J Med Sci 62: 167-175, 2008.

9. De Nunzio C, Miano R, Trucchi A, Finazzi Agrò E and Tubaro A: Finasteride for prostatic disease: an updated and comprehensive review. Expert Opin Drug Metab Toxicol 4: 1561-1568, 2008.

10. Steers WD: 5alpha-reductase activity in the prostate. Urology 58 (6 Suppl 1): 17-24, 2001.

11. Guess HA, Heyse JF and Gormley GJ: The effect of finasteride on prostatic-specific antigen in men with benign prostatic hyperplasia. Prostate 22: 31-37, 1993.

12. Bullock TL and Andriole GL Jr: Emerging drug therapies for benign prostatic hyperplasia. Expert Opin Emerg Drugs 11: 111-123, 2006.

13. Jeon GC, Park MS, Yoon DY, Shin CH, Sin HS and Um SJ: Antitumor activity of spinasterol isolated from Pueraria roots. Exp Mol Med 37: 111-120, 2005.

14. Klein Jr LC, Gandolfi RB, Santin JR, Lemos M, CechinelFilho V and de Andrade SF: Antiulcerogenic activity of extract, fractions, and some compounds obtained from Polygala cyparissias St. Hillaire \& Moquin (Polygalaceae). Naunyn-Schmiedebergs Arch Pharmacol 381: 121-126, 2010.

15. Villaseñor IM and Domingo AP: Anticarcinogenicity potential of spinasterol isolated from squash flowers. Teratog Carcinog Mutagen 20: 99-105, 2000.

16. Zhou CC, Sun XB, Liu JY, Luo SQ and Lu CY: Anti-inflammatory effect of alpha-spinasterol. Acta Pharmaceutica Sinica 20: 257-261, 1985 (In Chinese).

17. Maggi CA, Manzini S, Giuliani S and Meli A: Infravesical outflow obstruction in rats: A comparison of two models. Gen Pharmacol 20: 345-349, 1989.

18. Scolnik MD, Servadio C and Abramovici A: Comparative study of experimentally induced benign and atypical hyperplasia in the ventral prostate of different rat strains. J Androl 15: 287-297, 1994.

19. Van Coppenolle F, Le Bourhis X,Carpentier F,Delaby G, Cousse H, Raynaud JP, Dupouy JP and Prevarskaya N: Pharmacological effects of the lipidosterolic extract of Serenoa repens (Permixon) on rat prostate hyperplasia induced by hyperprolactinemia: comprison with finasteride. Prostate 43: 49-58, 2000.

20. VeereshBabu SV, Veeresh B, Patil AA and Warke YB: Lauric acid and myristic acid prevent testosterone induced prostatic hyperplasia in rats. Eur J Pharmacol 626: 262-265, 2010.

21. Miller $\mathrm{J}$ and Tarter TH: Combination therapy with dutasteride and tamulosin for the treatment of symptomatic enlarged prostate. Clin Interv Aging 4: 251-258, 2009.

22. Andriole G, Bruchovsky N, Chung LW, Matsumoto AM, Rittmaster R, Roehrborn C, Russell D and Tindall D: Dihydrotestosterone and the prostate: the scientific rationale for 5alpha-reductase inhibitors in the treatment of benign prostatic hyperplasia. J Urol 172: 1399-1403, 2004. 